\title{
Corrigendum to "Decomposing locally compact groups into simple pieces" [Math. Proc. Camb. Phil. Soc. 150 (1) (2011) 97-128]
}

\author{
BY PIERRE-EMMANUEL CAPRACE $\dagger$
}

UCLouvain, 1348 Louvain-la-Neuve, Belgium.

e-mail: pe.caprace@uclouvain.be

AND NICOLAS MONOD $\$$

EPFL, 1015 Lausanne, Switzerland.

e-mail: nicolas.monod@epfl.ch

(Received 9 February 2015; revised 7 August 2017)

Phillip Wesolek (personal communication) pointed out an error in Proposition 2.6 from our paper [1]; we thank him for his perspicacious remark, as well as for helpful comments on an earlier version of this correction. That error is corrected below; as we shall see, it only affects the statements of Theorem B and Proposition 2.6. An independent inaccurracy in [1, Theorem E(ii)], pointed out to us by Alain Valette (personal communication), is also rectified below. All other results of the original paper hold without change.

We retain all the notation and conventions from [1]; moreover any numbered statement quoted here refers to the corresponding statement from loc. cit. The invalid claim is that the intersection $\bigcap \mathscr{N}$ is trivial, in the last sentence of the proof of Proposition 2.6. That proposition must be replaced by the following:

PROPOSITION 2.6 (corrected). Let $G$ be a compactly generated totally disconnected locally compact group without non-trivial compact or discrete normal subgroup. Then:

(i) every non-trivial closed normal subgroup contains a minimal one;

(ii) the set $\mathscr{M}$ of non-trivial minimal closed normal subgroups need not be finite, but the subset $\mathscr{M}_{\mathrm{na}}$ of its non-abelian elements is finite;

(iii) each abelian $M \in \mathscr{M}$ is topologically locally finite, hence contained in $\operatorname{Rad}_{\mathscr{L} \mathscr{F}}(G)$;

(iv) for any proper subset $\mathscr{E} \subseteq \mathscr{M}$, the subgroup $\overline{\langle M \mid M \in \mathscr{E}\rangle}$ is properly contained in $G$.

In particular if $\operatorname{Rad}_{\mathscr{L F}}(G)=1$, then $\mathscr{M}=\mathscr{M}_{\mathrm{na}}$ is finite.

Assertions (i) and (iv) are stated and correctly proved in [1]. The erroneous assertion in loc. cit. is that the whole set $\mathscr{M}$ is finite. Indeed, a counter-example is provided by the group $\left(\mathbf{Q}_{p}\right)^{n} \rtimes \mathbf{Z}$, where a generator of the cyclic factor acts on the vector space $\left(\mathbf{Q}_{p}\right)^{n}$ by scalar multiplication by $p$. One checks that $G$ satisfies all hypotheses of the proposition. However, every one-dimensional subspace of $\left(\mathbf{Q}_{p}\right)^{n}$ is a minimal closed normal subgroup, and there are uncountably many such when $n>1$. Nevertheless, the original argument in the proof of Proposition 2.6 is correct provided no $M \in \mathscr{M}$ is abelian. In order to prove the more precise assertion (ii) above, we shall need the following subsidiary facts; the first one is well-known to the experts. 
LEMMA. A profinite group which is (topologically) characteristically simple is isomorphic to the direct product of copies of a given finite simple group.

Proof. Let $G$ be a characteristically simple profinite group, and $N$ be a maximal open normal subgroup. Then $G / N$ is a finite simple group, say $S$. The intersection of all $\operatorname{Aut}(G)$ conjugates of $N$ is trivial, which shows that $G$ injects continuously in a direct product $P$ of copies of $S$. Moreover the image of $G$ is dense, because $G$ maps onto each finite quotient of $P$, any such being a finite product of copies of $S$. Since $G$ is compact, that continuous injection is closed, hence a homeomorphism.

COROLLARY TO PROPOSITION 2.5. Let $G$ be a compactly generated totally disconnected locally compact group, and $\mathscr{N}$ be a filterling family of closed normal subgroups of $G$. Then there exist $N \in \mathscr{N}$ and a closed normal subgroup $Q$ of $G$ containing $B=\bigcap \mathscr{N}$ such that $Q / B$ is compact and $N / N \cap Q$ is discrete.

Proof. We apply Proposition $2 \cdot 5$ to the quotient group $G / B$. This yields a closed normal subgroup $Q$ of $G$, containing $B$ as a cocompact subgroup, and such that any filtering family of non-discrete closed normal subgroups of $G / Q$ has a non-trivial intersection. Since $Q / B$ is compact, the canonical projection $G / B \rightarrow G / Q$ is proper. Therefore each $N \in \mathscr{N}$ has closed image in $G / Q$, and the collection $\{N Q / Q\}_{N \in \mathscr{N}}$ forms a filtering family of closed normal subgroups of $G / Q$. By construction, the intersection $\bigcap\{N / B\}_{N \in \mathscr{N}}$ is trivial in $G / B$. Since the projection $G / B \rightarrow G / Q$ has compact kernel, it follows that the intersection $\bigcap\{N Q / Q\}_{N \in \mathscr{N}}$ is trivial in $G / Q$. Thus Proposition 2.5 ensures that $N Q / Q$ is discrete for some $N \in \mathscr{N}$. Equivalently $N / N \cap Q$ is discrete, as required.

Proof of Proposition 2.6 (corrected). As mentioned above, we only need to prove (ii) and (iii). For (iii), observe that since every abelian $M \in \mathscr{M}$ is non-discrete, it contains a nontrivial compact open subgroup, so that $\operatorname{Rad}_{\mathscr{L} \mathscr{F}}(M)$ is a non-trivial closed characteristic subgroup of $M$. Hence $\operatorname{Rad}_{\mathscr{L} \mathscr{F}}(M)$ is normal in $G$, so that $\operatorname{Rad}_{\mathscr{L} \mathscr{F}}(M)=M$ by minimality of $M$. In particular $\mathscr{M}=\mathscr{M}_{\text {na }}$ if $\operatorname{Rad}_{\mathscr{L} \mathscr{F}}(G)=1$.

For (ii), we set $N_{\mathscr{E}}=\overline{\langle M \mid M \in \mathscr{E}\rangle}$ for each non-empty $\mathscr{E} \subseteq \mathscr{M}$. By minimality, any two distinct elements of $\mathscr{M}$ have trivial intersection, hence they commute. In particular $N_{\mathscr{E}}$ and $N_{\mathscr{F}}$ commute as soon as the subsets $\mathscr{E}, \mathscr{F} \subseteq \mathscr{M}$ are disjoint.

We next remark that the family $\left\{N_{\mathscr{M}_{\mathrm{n}} \mid \mathscr{F}}\right\}$, where $\mathscr{F}$ ranges over the set of finite subsets $\mathscr{F} \subseteq \mathscr{M}_{\text {na }}$, is filtering and consists of non-discrete closed normal subgroups of $G$. Therefore, if $\bigcap_{\mathscr{F}} N_{\mathscr{M}_{\mathrm{n}} \mid \mathscr{F}}$ is trivial, then $\mathscr{M}_{\text {na }}$ must be finite as a consequence of Proposition $2 \cdot 5$. Thus (ii) holds in that case.

We now assume toward a contradiction that $\mathscr{M}_{\text {na }}$ is infinite. Thus $B=\bigcap_{\mathscr{F}} N_{\mathscr{M}_{\text {na }} \backslash \mathscr{F}}$ is non-trivial by the preceding paragraph.

By the Corollary to Proposition 2.5 above, there is a closed normal subgroup $Q$ of $G$ and a finite subset $\mathscr{F} \subseteq \mathscr{M}_{\text {na }}$ such that $Q / B$ is compact and $N / N \cap Q$ is discrete, where

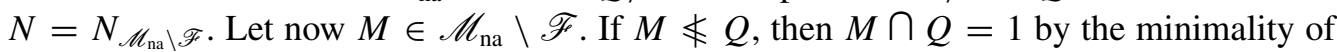
$M$, so that $M \leqslant N$ injects continuously in the discrete quotient $N / N \cap Q$. This implies that $M$ is discrete, in contradiction with the hypotheses. It follows that $N \leqslant Q$. In particular the quotient group $N / B$ is compact. 
Since $B \leqslant N_{\mathscr{M}_{\mathrm{na}} \backslash\{M\}}$ for each $M \in \mathscr{M}_{\mathrm{na}}$, we see that $B$ commutes with $M$. In particular $B$ is contained in the centre of $N$, so that the centre of $N$ is cocompact. Let now $U$ be a compact open subgroup of $N$. Then the normaliser $\mathscr{N}_{N}(U)$ is open (because it contains $U$ ) and cocompact in $N$ (because it contains the centre of $N$ ), hence it is of finite index. Therefore $U$ has only finitely many conjugates in $N$, and the intersection of all of them, say $V$, is a compact open normal subgroup of $N$. The quotient $N / V$ is discrete and generated by the images of the groups $M \in \mathscr{M}_{\mathrm{na}} \backslash \mathscr{F}$. Moreover $N / V$ has a non-trivial centre, since otherwise $B$ would be contained in $V$, and would thus be a non-trivial compact normal subgroup of $G$, contradicting the hypotheses. Thus there exist subgroups $M_{0}, \ldots, M_{n} \in$ $\mathscr{M}_{\mathrm{na}} \backslash \mathscr{F}$ and elements $x_{i} \in M_{i}$ such that the product $x_{0} x_{1} \ldots x_{n}$ is a non-trivial central element of $N / V$. We may assume that $x_{0}$ has non-trivial image. Since the groups $M \in \mathscr{M}_{\text {na }} \backslash$ $\mathscr{F}$ commute pairwise, it follows that $x_{0}$ is projected into the centre of the image of $M_{0}$. Those observations show that $M_{0} \in \mathscr{M}_{\text {na }} \backslash \mathscr{F}$ admits a discrete quotient with a non-trivial centre.

The final contradiction will now be obtained by showing that, on the other hand, for all $M \in \mathscr{M}_{\text {na }} \backslash \mathscr{F}$, every discrete quotient of $M$ is centrefree. In order to establish that, we first recall that $\overline{B M} / B$ is compact, since $N / B$ is so. Since $B$ commutes with $M$, this implies that $\overline{M \mathscr{Z}_{G}(M)} / \mathscr{Z}_{G}(M)$ is compact.

Let $\pi: G \rightarrow G / \mathscr{Z}_{G}(M)$ be the canonical projection. The restriction of $\pi$ to $M$ is injective, since otherwise the minimality of $M$ would imply that $M \leqslant \mathscr{Z}_{G}(M)$, so $M$ would be abelian, a contradiction.

We next claim that the compact group $\overline{\pi(M)}=\overline{M \mathscr{Z}_{G}(M)} / \mathscr{Z}_{G}(M)$ is a minimal closed normal subgroup of the quotient $G / \mathscr{Z}_{G}(M)$. Indeed, given any non-trivial closed normal subgroup of $G / \mathscr{Z}_{G}(M)$, its preimage in $G$ is a closed normal subgroup $P$ which does not commute with $M$. Thus $P \cap M \geqslant[P, M]$ is non-trivial, so that $M \leqslant P$ by the minimality of $M$. This confirms that $P / \mathscr{Z}_{G}(M)$ contains $\overline{\pi(M)}$.

Being a minimal closed normal subgroup, the profinite group $\overline{\pi(M)}$ is (topologically) characteristically simple. By the Lemma above, we see that $\overline{\pi(M)}$ is the direct product of copies of a finite simple group $S$, which must be non-abelian since $M$ is not abelian. Let then $T$ be a direct factor of $\overline{\pi(M)}$ isomorphic to $S$. Since $\pi(M)$ is dense and since $T$ is not abelian, we see that $\pi(M)$ does not commute with $T$. Since $T$ is simple, we infer that $T=[T, \pi(M)] \leqslant \pi(M)$. This implies that $\pi(M)$ contains the direct sum $D$ of all simple factors of $\overline{\pi(M)}$. Since $D$ is normalised by $G$ in $G / \mathscr{Z}_{G}(M)$, its preimage $M \cap \pi^{-1}(D)$ is a normal subgroup of $G$ contained in $M$, and isomorphic to $D$, because the projection of $M$ to $G / \mathscr{Z}_{G}(M)$ is injective. Denoting $M \cap \pi^{-1}(D)$ also by $D$, we see that $D$ is dense in $M$ by minimality. Since $D$ is a direct sum of a copies of a non-abelian finite simple group, any quotient of $D$ is also of that form, and is in particular centrefree. Since $D$ is dense in $M$, it surjects onto any discrete quotient of $M$, thereby confirming the claim that every discrete quotient of $M$ is centrefree. This contradiction concludes the proof of (ii).

Proposition 2.6 was used in an essential way in the proof of Theorem B, whose statement must therefore be amended as follows.

THEOREM B (corrected). Let $G$ be a compactly generated locally compact group. Then one of the following holds:

(i) $G$ has an infinite discrete normal subgroup;

(ii) $G$ has a non-trivial closed normal subgroup which is compact-by-\{connected soluble\}; 
(iii) $G$ has non-trivial minimal closed normal subgroups, of which only finitely many are non-abelian.

The corrected Theorem B follows from the corrected Proposition 2.6 by the same argument as in [1]: it suffices to observe that the correspondence between the closed normal subgroups of $G$ contained in $D$ and the closed normal subgroup of $G / G_{1}$ contained in $D G_{1} / G_{1}$ established along the proof preserves abelian subgroups.

We now review all the other places in [1] where Proposition 2.6 (or Theorem B) was used. In the proofs of Theorem C, Corollary D and Theorem E, the finiteness claim of Proposition 2.6 is applied to a group whose LF-radical is trivial, so that the arguments hold without change. The only other place where Proposition 2.6 was invoked is in the proof of Proposition II. 1 from Appendix II. There again, no further correction is needed since the ambient group also has trivial LF-radical. Indeed, as observed in that proof, the group $G$ injects continuously into the product of non-compact, compactly generated topologically simple groups $\prod_{K \in \text { Max }} G / K$. Since each simple quotient $G / K$ has trivial LF-radical, the same holds for $G$.

We take this opportunity to rectify another inaccurracy in [1]. In [1, Theorem E(ii)], the first sentence claiming that $G$ be monolithic with monolith $L \cong \mathbf{R}^{n}$ must be replaced by the weaker assertion that $G$ has a cocompact closed normal subgroup $L \cong \mathbf{R}^{n}$. All other parts of the statement, including the description of $G / L$, hold. The arguments given in the proof of Theorem $\mathrm{E}$ are correct, but they do not establish the monolithicity of $G$ in case (ii). As remarked by Alain Valette, this conclusion may indeed fail: the group $G=\mathbf{R}^{2} \rtimes S_{3}$, where the symmetric group $S_{3}$ acts via an irreducible representation, satisfies all the hypotheses of the theorem, but it is not monolithic since it has a chain of discrete cocompact normal subgroups with trivial intersection.

\section{REFERENCE}

[1] P.-E. CAPrace and N. Monod. Decomposing locally compact groups into simple pieces. Math. Proc. Camb. Philo. Soc. 150 (2011), no. 1, 97-128. 